\title{
Research on the Finger Knuckle Print Recognition Method Based on Improved Canny Operator
}

\author{
Xiufeng Zhang, Wei Wang, Qiang Ding, Tianyi Ma and Chunyang Hao \\ College of Mechanical and Electrical Engineering, Dalian Minzu University, Liao Ning, China \\ ${ }^{*}$ Corresponding author
}

\begin{abstract}
In order to improve the accuracy of the classification and classification of the finger knuckle print, a method based on the improved Canny operator for the edge detection of the Finger Knuckle Print image is proposed. Firstly, the region of interest (ROI) of the Finger Knuckle Print is positioned. Secondly, The method optimized the edge detection of the finger knuckle print images from two aspects: traditional Canny algorithm filtering, gradient direction. For the poor performance of Gauss filter used by traditional Canny algorithm in limitation of removing gauss noise and loss of edge details, Adaptive median filtering is used instead of Gaussian filtering for filtering. For the problem that the Canny algorithm is easy to detect the false edge, the edge was refined by adding the direction gradient template in the process of calculating the gradient direction of the image. Finally, information entropy is used to illustrate the effectiveness of the algorithm. Compared with traditional operators, this method has better stability and robustness.
\end{abstract}

Keywords-canny; the finger knuckle print; ROI; daptive median filtering

\section{INTRODUCTION}

Image segmentation is an important part of image feature extraction, and it is one of the hotspots and difficulties in the field of biometrics. Image segmentation is a process of extracting regions of interest from an image[1,2]. Common segmentation methods are: edge, threshold, region, specific theory, and so on. Traditional edge detection algorithms have inadequacies in application: Sobel edge detection algorithms positioning is not accurate; Robert algorithms positioning is more accurate, but sensitive to noise; Prewit uses local gray average, easy to detect false edges and causes oversegmentation; the LOG filter has a large loss of detail on the image and low edge precision. In this paper, the improved Canny operator is used to detect the Finger Knuckle Print, which can effectively avoid the problems of the above algorithms.

Duan Hongyan used bilateral filtering instead of traditional Gaussian filtering to control the weight parameters of bilateral filters and used wavelet transform to reduce the low-frequency coefficients of the image amplification high-frequency coefficients and enhance the image details. Liu Lixia used guided filtering instead of Gaussian filtering to maintain image edge characteristics. Li Baolei et al. use Otsu algorithm instead of double threshold algorithm to choose threshold independently to reduce noise.the finger knuckle print has the advantages of convenient collection, clear texture information and easy extraction, It has a good application prospect. Some researchers have studied the ROI segmentation and obtained some research results. In this paper, the image ROI (Region Of Interest region) is extracted, and the image is edge-extracted by the improved Canny operator. The method in this paper can achieve the extraction of effective regions to reduce redundancy, and has better edge retention effect, which has certain practical value.

\section{POSITIONING FINGER KNUCKLE PRINT AREA}

In the process of image processing of the Finger Knuckle Print, only the part with the richest texture information is processed, which is called the ROI of the region of interest, and the other parts are not considered. In this paper, the finger contour is used to realize the ROI segmentation of the Finger Knuckle Print image.

For the extraction of the finger contour, we use a $3 \times 3$ matrix $\left[\begin{array}{lll}-1 & 0 & 1 \\ -2 & 0 & 2 \\ -1 & 0 & 1\end{array}\right]$ for detecting the edge of the finger.

The position of the finger in the ingested picture is fixed. The width of the effective area can be determined according to the maximum coordinate and the minimum coordinate in the extracted contour of the image. The length of the effective area is confirmed and the rectangular area can be determined by the width and the length. This area is (ROI). Because different people's finger thickness is different, we need to determine a standardized ROI area, using bilinear interpolation for size normalization, for the standard effective area size setting $96 \times 64$, as shown in Figure I.

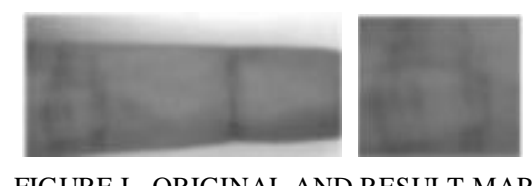

\section{IMPROVED CANNy EDGE DETECTION SEGMENTATION ALGORITHM}

\section{A. The Flow of Traditional Canny Algorithm}

The basic flow of the Canny edge detection algorithm is shown in Figure II.[6,7,8] Firstly, Gaussian filtering is performed on the input image, then the first-order differential calculation is performed on the image to perform edge 


$$
\mathrm{A}: A 1=Z_{\text {med }}-Z_{\text {min }}, A 2=Z_{\text {med }}-Z_{\text {max }}
$$

detection on the image. Next, different gradient templates are used for non-maximum suppression. However, the first-order differential calculation method only considers horizontal and vertical,The direction causes the image information to be incomplete. The final step is to perform edge connection based on the image processed by the high and low thresholds, and then perform edge detection by suppressing the isolated weak edges.

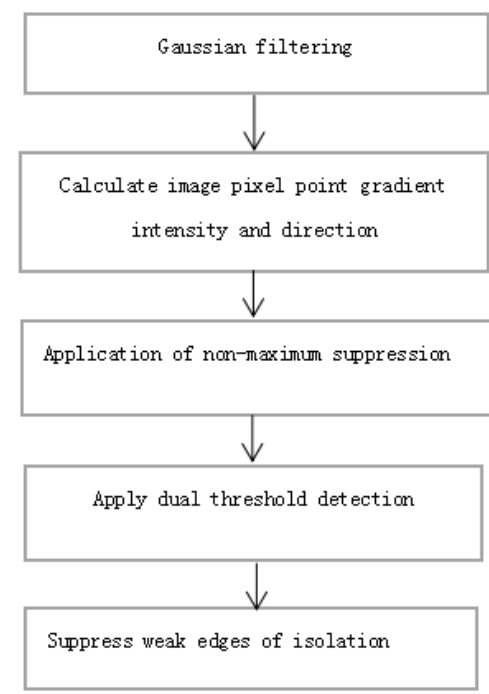

FIGURE II. CANNY EDGE DETECTION ALGORITHM PROCESSING FLOW

\section{B. Canny Algorithm Improvement}

a) Gaussian filtering blurs the texture information while smoothing the the Finger Knuckle Print image. In order to segment the image more accurately, this paper chooses adaptive median filtering instead of Gaussian filter, which can effectively protect the details of the knuckle pattern. And dynamically change the window size of the median filter to take into account the effects of noise removal and detail protection. Pre-defined symbols and Specific steps are as follows.

$S_{x y}$ : the active area of the filter;

$Z_{\min }: S_{x y}$ Minimum gray value;

$Z_{\max }: S_{x y}$ Maximum gray value;

$Z_{\text {med }} S_{x y}$ The median of all gray values;

$Z_{x y}$ : Indicates the gray value of the original image;

$S_{\max }: S_{x y}$ The maximum window size allowed;

Traditional adaptive median filtering is divided into two phases, $\mathrm{A}$ and $\mathrm{B}$ :
If $A 1>0$ and $A 2<0$, jump to $\mathrm{B}$, otherwise increase the window size. If the increased size is $\leq S_{\max }$, then A is repeated. Otherwise, output $Z_{\text {med }}$ directly.

$$
\mathrm{B}: B 1=Z_{x y}-Z_{\min }, B 2=Z_{x y}-Z_{\max }
$$

If $B 1>0$ and $B 2<0$, then the output is $Z_{x y}$, otherwise the output is $Z_{\text {med }}$.

Compared with Gaussian filtering, the adaptive median filter could highlights the texture information of the knuckles while smoothing the image for the next step.

b) The traditional Canny algorithm calculates the magnitude and direction using a $(2 \times 2)$ template.

$$
\begin{gathered}
G=\sqrt{G_{x}^{2}+G_{y}^{2}} \\
\theta=\arctan \left(G_{y} / G_{X}\right)
\end{gathered}
$$

The traditional Canny operator is sensitive to noise and cannot detect edges very efficiently. so, the detection algorithm adds two directions of 45 degrees and 135 degrees on the basis of horizontal and vertical directions to calculate the first-order partial guide. The formula for calculating the gradient value is as follows:

Horizontal gradient

$$
G_{x}(x, y)=f(x+1, y)-f(x-1, y)
$$

Vertical gradient

$$
G_{y}(x, y)=f(x, y+1)-f(x, y-1)
$$

45 degree gradient

$$
G_{45}(x, y)=f(x-1, y+1)-f(x+1, y-1)
$$

135 degree gradient

$$
G_{135}(x, y)=f(x+1, y+1)-f(x-1, y-1)
$$

The current pixel gradation is worth the formula (9) (10) corresponding to the gradient magnitude $\mathrm{G}(\mathrm{i}, \mathrm{j})$ and the gradient angle $\theta(x, y)$.

$$
G(x, y)=\sqrt{G x(x, y)^{2}+G y(x, y)^{2}+G_{45}(x, y)^{2}+G_{135}(x, y)^{2}}
$$




$$
\theta(x, y)=\arctan \left(\frac{\mathrm{Gy}(\mathrm{i}, \mathrm{j})}{G x(i, j)}\right)
$$

The pixel gradient is calculated by 8 directions, which makes the image positioning more accurate, and also greatly reduces the false detection rate and the missing edge.

c) Non-maximum value suppression is a very important technique for edge processing. When the image is subjected to gradient calculation, the image is very blurred. In order to obtain standard edge information, the local maximum value can be realized by non-maximum value suppression. All gradient values outside the suppression are zero. The algorithm principle is to compare the current pixel value with the pixels in other different directions. If the current pixel value is greater than other, the pixel will remain, otherwise the pixel will be suppressed.

To increase accuracy, linear interpolation is used to compare pixel gradients between two adjacent pixels across the gradient direction. Specifically, as shown in Figure III.

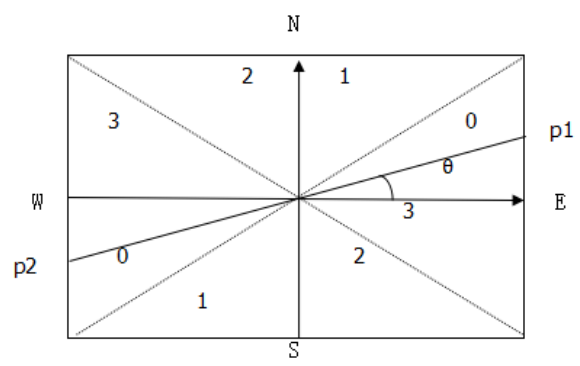

FIGURE III. THE SEGMENTATION OF THE GRADIENT DIRECTION

The gradient direction of the pixel $\mathrm{P}$ is $\theta$, and the gradient linear interpolation of the pixel $\mathrm{P} 1$ and $\mathrm{P} 2$ is:

$$
\begin{gathered}
\tan (\theta)=\frac{G y}{G x} \\
G_{P 2}=(1-\tan (\theta)) \times W+\tan (\theta) \times S W
\end{gathered}
$$

d) In order to realize that the remaining pixels represent the actual edge more accurately, the image is subjected to double threshold detection. Since some color and noise changes cause some edge pixels, the enhancement of edge pixels with weak gradient values can reduce unnecessary interference and preserve the edge pixel values of high gradient values. A strong edge pixel if the edge pixel gradient value is greater than the high threshold, a weak edge pixel if the gradient value is between the high and low thresholds, suppressing the pixel if the pixel gradient value is less than the low threshold.

e) This step needs to suppress isolated low threshold points. For weak edge pixels, the main cause of these pixels is caused by changes in color and noise when extracting edges from images. In order to make the result more accurate, the weak edge should be suppressed. The weak edge caused by the real edge is connected to the strong edge pixel. In order to track the edge connection, by finding weak edge pixels and 8 neighborhood pixels, weak edge pixels can be retained if strong edge pixels are found, otherwise they will be removed.

\section{EXPERIMENTAL RESULTS AND ANALYSIS}

The experimental environment of this paper is: CPU is AMD Athlon processor, memory is $2.75 \mathrm{G}$ operating system is Window, and the programming environment used is MATLAB2016A.

In order to prove the validity of the paper, two images are selected for experiment and compared with other edge detection operators and the segmentation results of traditional edge detection canny operators. As shown in Fig. IV, it can be found that the segmentation effect of the improved algorithm is more ideal than the traditional algorithm.

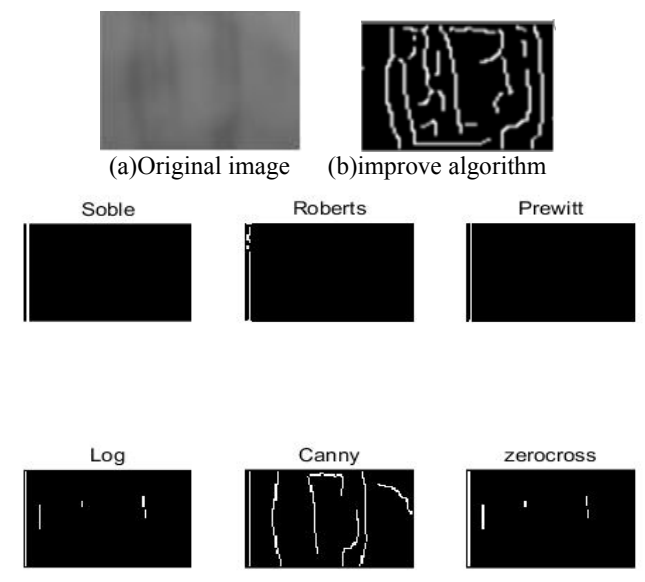

(c)Traditional algorithm

FIGURE IV. COMPARISON OF RESULTS BETWEEN TRADITIONAL AND IMPROVED ALGORITHMS

In the process of image processing, it is necessary to discriminate the sharpness of an image and the pros and cons of the image segmentation result, thus leading to the concept of information entropy. Most of the time, it is used as a quantitative standard for evaluating images. So this paper quotes the concept of information entropy to illustrate the effectiveness of the improved algorithm, as shown in Table 1.

TABLE I. COMPARISON OF INFORMATION ENTROPY

\begin{tabular}{|c|c|c|c|c|c|c|}
\hline algorithm & $\begin{array}{c}\text { Traditional } \\
\text { Canny }\end{array}$ & Log & Prewitt & Sobel & $\begin{array}{c}\text { Robert } \\
\text { s }\end{array}$ & $\begin{array}{c}\text { Impro } \\
\text { ve } \\
\text { Canny }\end{array}$ \\
\hline $\begin{array}{c}\text { Informatio } \\
\text { n entropy }\end{array}$ & 0.368 & 0.103 & 0.235 & 0.228 & 0.173 & 0.6329 \\
\hline
\end{tabular}

In this paper, 50 images are selected to compare with the improved Canny operator and the traditional Canny operator. The results are shown in Fig. V. 
[7] Wang Baojun, Zhao Haiqing, Liu Chao.Canny operator improved edge detection algorithm[J].Science and Technology Innovation,2018(27):1314.

[8] Guohua Wu, Dongyue Yang,Chen Chang, Longfei Yin, Bin Luo, Hong Guo. Optimizations of Canny Edge Detection in Ghost Imaging[J]

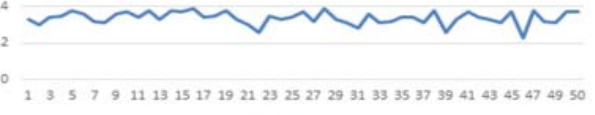
—Cann

(a)Traditional Canny information entropy

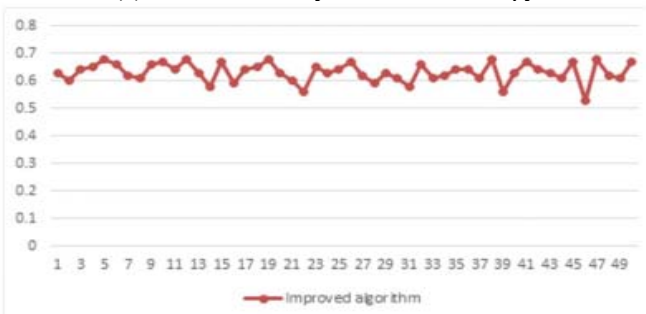

(b)Improve Canny Information Entropy

FIGURE V. COMPARISON OF INFORMATION ENTROPY TEST RESULTS

\section{CONCLUSION}

In this paper, an edge detection method for the Finger Knuckle Print image based on the improved traditional Canny operator is proposed for the edge detection problem of the Finger Knuckle Print image. This method improves the two aspects of the traditional Canny operator: (1) using adaptive median filtering instead of Gaussian filtering to smooth the image; (2) adding two in the process of calculating the gradient direction by the traditional Canny operator The gradient template of the direction; the method not only optimizes the denoising performance, but also ensures the accuracy of the edge connection, so that the edge detection of the knuckle image is better. Of course, the algorithm in this paper occasionally has a poor edge segmentation connection in the image edge detection process with partial background, which will be further improved in the subsequent research.

\section{REFERENCES}

[1] Ghaleh S R, Ahmadi K S, Kheradmand R, et al.Improved edge detection in computational ghostimaging by introducing orbital angular momentum[J].Applied optics,2018,57(32):9609-9614

[2] Kolda Lukas, Krejcar Ondrej, Selamat Ali, Kuca Kamil, Fadeyi Oluwaseun. Multi-Biometric System Based on Cutting-Edge Equipment for Experimental Contactless Verification.[J]. Sensors (Basel, Switzerland),2019,19(17).

[3] Duan Hong-yan, Shao Hao, Zhang Shu-zhen, Zhang Xiao-yu, Wang Xiao-hong. An Improved Image Edge Detection Algorithm Based on Canny Operator[J].Journal of Shanghai Jiaotong University,2016,50(12):1861-1865.

[4] Liu Lixia, Li Baowen, Wang Yangping, Yang Jingyu. Improvement of remote sensing image segmentation based on Canny edge detection[J].Computer Engineering and Applications,2019,55(12):54$58+180$.

[5] Li Baolei, Li Qingying, Qin Yukun, Liu Yunfei, Xu Ensong, Wang Xiaowei.An Improved Canny Thermal Infrared Edge Detection Method[J].Computer Age,2019(01):63-66.

[6] Zhang Yueyuan, Zeng Qinghua, Liu jianye, et al. Canny based improved image edge detection algorithm[J]. Navigation and Control,2019,18(1):84-90. 